\title{
Assessing the necessity of chimpanzee experimentation
}

On December 15, 2011, the US Institute of Medicine (IOM) released Chimpanzees in Biomedical and Behavioral Research: Assessing the Necessity (Altevogt et al., 2011a). The report responded to a National Institutes of Health (NIH) request for expert guidance on the scientific need to use chimpanzees within such research.

The IOM committee concluded that most current use of chimpanzees for biomedical research - including RSV vaccine, therapeutic hepatitis $\mathrm{C}(\mathrm{HCV})$ vaccine, and $\mathrm{HCV}$ antiviral drug development - is not warranted, with the possible exception of two very limited research uses: for the production of monoclonal antibodies (mAbs) and the development of prophylactic $\mathrm{HCV}$ vaccines. The committee stated, "it will be very difficult to defend the necessity of nearly all current biomedical research on chimpanzees." Yet it stopped short of recommending an outright ban (Altevogt et al., 2011b).

Even this limited support of invasive chimpanzee research is questionable, however. The committee noted that, "Production of monoclonal antibodies after immunization in other species or through in vitro synthetic methods is equally powerful for the generation of such reagents." They also noted four methods currently in use that lessen the need for safety tests of mAbs in chimpanzees. However, they still supported the use of chimpanzees for mAbs developed using older technologies, although they expected such use would cease within five years. Yet, it would seem far more ethical, and could also potentially yield safety, efficacy and financial benefits, if the most modern technologies were used without further delay. As Bettauer (2011) noted with respect to chimpanzee use in $\mathrm{mAb}$ research and drug development, "Available alternatives, together with ethical and economic reasons, suggest that the use of the chimpanzee in this manner may not be necessary or appropriate."

The committee noted that HCV antiviral drug and therapeutic vaccine development does not require the use of chimpanzees, but were uncertain about their necessity for the development of prophylactic vaccines. However, they also noted the existence of alternative research strategies: "studies in consenting individuals at high risk for natural HCV infection can be ethically done provided that these vaccines are first shown to be safe and immunogenic in experimental animals, such as mice and nonhuman primates." After reviewing 109 chimpanzee HCV studies, Bettauer (2010) found considerable problems with statistical validity, repeatability, and the biological relevance of the chimpanzee model. Bailey (2010) similarly found that, "claims of the necessity of chimpanzees in historical and future hepatitis $C$ research are exaggerated and unjustifiable, respectively." $\mathrm{He}$ concluded that, "Unfounded claims of its necessity should not discourage changes in public policy regarding the use of chimpanzees in US laboratories."

Although the committee failed to identify any current biomedical research field in which invasive chimpanzee studies are definitely necessary, it concluded that, " $a$ new, emerging, or reemerging disease or disorder may present challenges to treatment, prevention, and/or control that defy non-chimpanzee models and technologies and thus may require their future use. Therefore, an outright ban on biomedical chimpanzee research would not be appropriate."

In reaching this conclusion, however, the committee made very little mention of the profound animal welfare and ethical problems raised by invasive chimpanzee research. The advanced cognitive, sociological and related characteristics of chimpanzees render their use particularly ethically problematic. Such research is also particularly costly. Accordingly, the concrete benefits of such research - particularly in advancing human healthcare - must be particularly substantial, probable and independently verifiable, in order for it to be considered ethically and fiscally justifiable. Yet the relevant systematic reviews published to date suggest that this is far from the case. The contributions of invasive chimpanzee research to biomedical progress appear highly questionable, and it rarely, if ever, makes important contributions to clinical interventions efficacious in human patients (Bailey, 2008, 2009, 2010; Bettauer, 2010, 2011; Knight, 2007, 2008).

This is why almost all nations that have considered invasive chimpanzee experimentation have implemented policy or legislative bans on such research, with the exception of noninvasive 
observational or behavioral research, or research conducted at ensuring the survival of the individual in question, or of the species. Only the US, and possibly Gabon - whose status is unclear - still persist with invasive chimpanzee research.

The US should utilize the opportunity afforded by the Great Ape Protection and Cost Savings Act of 2011 to similarly ban invasive chimpanzee experimentation. This would not require the banning of all chimpanzee research, however. Clear-cut examples include non-invasive observational or behavioral studies of free-living or sanctuary chimpanzees, and experimental treatment of chimpanzees genuinely suffering from severe, naturally occurring disease or injury, when conventional treatment is ineffective. Rigorous implementation of policies such as these would restore to chimpanzee research the balance between human and animal interests expected by society, and demanded by detailed ethical review.

Instead however, the committee concluded that invasive chimpanzee research would be acceptable if it met certain criteria: the knowledge gained must be necessary to advance public health; the research cannot ethically be conducted on a human being, and is impossible using other species or inanimate research tools; and the chimpanzees used must be maintained in natural habitats or in physical and social environments that are similarly ethologically appropriate. Additionally, forgoing the use of chimpanzees for the research in question must significantly slow or prevent important advancements to prevent, control, and/or treat life-threatening or debilitating conditions. For studies of comparative genomics and behavioral research, the criteria were that studies must provide otherwise unobtainable insights into comparative genomics, normal and abnormal behavior, mental health, emotion, or cognition; and that all experiments must be performed on acquiescent animals (who participate voluntarily, without coercion), using techniques that are, at most, minimally invasive, painful or distressing.

NIH Director Francis Collins accepted the committee's recommendations. The NIH announced that until it issues further policy implementing these recommendations, it will not fund any new or competing projects (that is, renewals and revisions) involving chimpanzee research; nor will it allow any new projects to proceed using NIH owned or supported chimpanzees (NIH, 2011). A project-by-project review will also be conducted to determine whether ongoing research fits the recommended criteria. Dr Collins estimated that about 37 research projects might be affected, of which perhaps half could be discontinued (Anon., 2011; Collins, 2011).

\section{References}

Altevogt, B. M., Pankevich, D. E., Shelton-Davenport, M. K., and Kahn, J. P. (eds.) (2011a). Chimpanzees in biomedical and behavioral research: Assessing the necessity. Washington DC, USA: National Academies Press. http://iom.edu/ chimpstudy (accessed 20.12.2011).

Altevogt, B. M., Pankevich, D. E., Pope, A. M., and Kahn, J. P. (2011b). Guiding limited use of chimpanzees in research. Science, Epub ahead of print 15 Dec. 2011. doi: 10.1126/science.1217521.

Anon. (2011). NIH to adopt strict new limits on using chimpanzees in medical research, saying most unneeded.The Washington Post, 15 Dec, 2011. http://www.washingtonpost.com/national/healthscience/major-science-group-says-chimpanzees-should-hardly-ever-be-used-for-medical-research-anymore/2011/12/15/ gIQAEcf5vO_story_1.html (accessed 15.12.2011).

Bailey, J. (2008). An assessment of the role of chimpanzees in AIDS vaccine research. ATLA 36, 381-428.

Bailey, J. (2009). An examination of chimpanzee use in human cancer research. ATLA 37, 399-416.

Bailey, J. (2010). An assessment of the use of chimpanzees in hepatitis $C$ research past, present and future: 1 . validity of the chimpanzee model. ATLA 38, 387-418.

Bettauer, R. H. (2010). Chimpanzees in hepatitis C virus research: 1998-2007. J. Med. Primatol. 39, 9-23.

Bettauer, R. H. (2011). Systematic review of chimpanzee use in monoclonal antibody research and drug development: 19812010. ALTEX 28, 103-116.

Collins, F. (2011). Statement by NIH Director Dr. Francis Collins on the Institute of Medicine report addressing the scientific need for the use of chimpanzees in research. NIH News. http://www.nih.gov/news/health/dec2011/od-15. htm (accessed 05.01.2012).

Knight, A. (2007). The poor contribution of chimpanzee experiments to biomedical progress. J. Appl. Anim. Welf. Sci. 10, 281-308.

Knight, A. (2008). The beginning of the end for chimpanzee experiments? Philos. Ethics Humanit. Med. 3, 16. http://www. peh-med.com/content/3/1/16

National Institutes of Health - NIH (2011). NIH research involving chimpanzees. http://grants.nih.gov/grants/guide/notice-files/NOT-OD-12-025.html (accessed 05.01.2012).

Andrew Knight, PhD, MRCVS, DipECAWBM-AWSEL, FOCAE Oxford Centre for Animal Ethics Oxford, UK

Phone: +44 7824376709

www.oxfordanimalethics.com/ who-we-are/fellows/ 